\title{
IMBEDDED SUBGROUPS OF ABELIAN GROUPS
}

\author{
W. P. BERLINGHOFF, J. D. MOORE and J. D. REID
}

(Received 8 April 88)

Communicated by $\mathbf{H}$. Lausch

\begin{abstract}
A subgroup $H$ of an abelian $p$-group $G$ is pure in $G$ if the inclusion map of $H$ into $G$ is an isometry with respect to the (pseudo-) metrics on $H$ and $G$ associated with their $p$-adic topologies. In this paper, those subgroups (called here imbedded subgroups) of abelian groups for which the inclusion is a homeomorphism with respect to the $p$-adic topologies are studied, the aim being to compare the concepts of imbeddedness and purity. Perhaps the main results indicate that imbedded subgroups are considerably more abundant than pure subgroups. Groups for which this is not the case are characterized.
\end{abstract}

1980 Mathematics subject classification (Amer. Math. Soc.) (1985 Revision): 20 K 10.

This paper seeks, in part, to repair a gap in the recent literature of abelian groups. A preliminary version of the paper, written by the second and third authors in 1970 and accepted for publication but never published, has been cited as "to appear" in several subsequent papers, including [6], [7] and [8]. Meanwhile, additional relevant results have been found; they are included in this expanded version. Although some of the underlying ideas of this paper can be treated in a more general group-theoretic context (as in [8]), the author feel that these ideas flow most naturally from specific consideration of the $p$-adic topology.

The subgroups of an abelian group $G$ that interest us are those subgroups $H$ for which the $p$-adic topology in $H$ coincides with the topology that $H$

Some of the results in this paper appear in the second author's Ph.D. dissertation, Syracuse University, 1969; others appear in the first author's Ph.D. dissertation, Wesleyan University, 1976. This work was supported in part by National Science Foundation Grants GP-5878 and GP-19351.

(C) 1990 Australian Mathematical Society $0263-6115 / 90 \$ A 2.00+0.00$ 
acquires as a subspace of $G$. This means that there should exist a function $l$ from the non-negative integers into themselves satisfying $H \cap p^{l(n)} G \subseteq p^{n} H$ for all $n$. Then $H$ is $p$-pure in $G$ if and only if the identity function serves, and the degree of complication of the function $l$ indicates the extent to which $H$ is not $p$-pure. Section 1 establishes some elementary general facts. In Section 2 we give some examples to show that the functions $l$ can be quite complicated. Section 3 discusses subgroups that are "almost pure." Section 4 relates the subgroups of interest to us with kernels of purity, and its results are applied in Section 5 to characterize the subgroups discussed in Section 3. Section 6 characterizes those groups $G$ all of whose imbedded subgroups are "almost pure". Finally, Section 7 settles a conjecture raised in [6]. All groups considered are abelian and our notation is that of [1] and [2].

\section{Imbedded subgroups}

Definitions 1.1. A subgroup $H$ of a group $G$ is imbedded (respectively $p$-imbedded) in $G$ provided there exists a function $l$ from the non-negative integers $\mathrm{N}$ into themselves such that $H \cap l(n) G \subseteq n H\left(H \cap p^{l(n)} G \subseteq p^{n} H\right)$ for all $n$. We call $l$ an imbedding function for $H$ in $G$ and write $H<G\left(H<_{p} G\right)$ to indicate its existence. If $l$ is an imbedding function for $H$ in $G$ we call $\delta$, defined by $n+\delta(n)=l(n)$, the corresponding difference function.

Clearly, if $l$ is an imbedding function for $H$ in $G$ and if $l^{\prime}$ is any function from $\mathrm{N}$ to $\mathrm{N}$ such that $l^{\prime}(n) \geq l(n)$ for all $n$, then $l^{\prime}$ is also an imbedding function for $H$ in $G$. Moreover, the collection of all imbedding functions for $H$ in $G$ contains its (pointwise) infimum, which we call the minimal imbedding function. It is also clear that $H<G$ if and only if $H<_{p} G$ for all primes $p$. For the relation between the global imbedding function and the local ones for an imbedded subgroup $H$ of $G$ we have

Proposition 1.2. Let $H<G$ with minimal function $l$. Then $l$ is multiplicative: if $(n, m)=1$, then $l(n m)=l(n) l(m)$.

Proof. The prime divisors $p$ of $l(n)$ must occur among those of $n$, for if $(p, n)=1$ and $l(n)=p^{e} s$, with $(s, p)=1$, let $x \in H \cap s G$. Then $p^{e} x \in$ $H \cap l(n) G \subseteq n H$ and, for suitable integers $a$ and $b$, $a n+b p^{e}=1$, so $x=$ $a n x+b p^{e} x \in n H$. Thus $H \cap s G \subseteq n H$, implying $s \geq l(n)$ by the minimality of $l$. This gives $e=0$, as desired.

Now suppose $(n, m)=1$. By the remarks above, this implies $(l(n), l(m))=$ 1 , so

$$
H \cap l(n) l(m) G=H \cap l(n) G \cap H \cap l(m) G \subseteq n H \cap m H=n m H,
$$


implying

$$
l(n m) \leq l(n) l(m) .
$$

For the opposite inequality, put $l(n m)=r s$, where $(r, m)=(s, n)=1$, and let $a s+b n=1$. Then, for $x \in H \cap r G$,

$$
s x \in H \cap l(n m) G \subseteq n m H \subseteq n H,
$$

so $x=a s x+b n x \in n H$. This yields $l(n) \leq r$. Similarly, $l(m) \leq s$, so

$$
l(n) l(m) \leq r s=l(n m) .
$$

COROLLARY 1.3. Let $H<G$ with minimal imbedding function l (p-imbedding functions $l_{p}$ ) and suppose $n=\prod_{i=1}^{k} p_{i}^{e_{i}}$ for distinct primes $p_{i}$. Then

$$
l(n)=\prod_{i=1}^{k} l\left(p_{i}^{e_{i}}\right) \quad \text { and } \quad l\left(p_{i}^{e_{i}}\right)=p_{i}^{l_{p_{i}}\left(e_{i}\right)}
$$

A number of elementary properties of imbeddedness are collected in the proposition below, whose proof is omitted. We state the local version, but this implies the global version. In the case of $p$-groups, and somewhat less successfully in general, the following lemmas reduce certain questions about imbedded subgroups to the case of direct sums of cyclic groups. Proposition 1.7 is one such example, which will be applied later. Of course, for $p$-groups, imbeddedness and $p$-imbeddedness coincide, so we omit the reference to $p$ in this case.

Proposition 1.4. Let $H$ and $K$ be subgroups of $G$ with $K \subseteq H$.

(i) If $K<_{p} G$, then $K<_{p} H$.

(ii) If $K<_{p} H$ and $H<_{p} G$, then $K<_{p} G$.

(iii) If $H<_{p} G$, then $H / K<_{p} G / K$.

(iv) If $K<_{p} G$ and $H / K<_{p} G / K$, then $H<_{p} G$.

(v) If $H<_{p} G$, then $H \cap p^{\omega} G=p^{\omega} H$.

Sometimes it is useful to know that in (ii) above, if $l_{1}$ and $l_{2}$ are imbedding functions for $H$ in $G$ and for $K$ in $H$, respectively, then the composite $l_{1} \circ l_{2}$ is an imbedding function for $K$ in $G$. Similarly, in (iv), if $l_{1}$ and $l_{2}$ are imbedding functions for $K$ in $G$ and for $H / K$ in $G / K$, respectively, then $l_{2} \circ l_{1}$ is an imbedding function for $H$ in $G$. Also, if $l$ is an imbedding function for $H$ in $G$, then $l(n) \geq n$ unless $p^{n-1} H$ is divisible. 
Lemma 1.5. Suppose that $H<_{p} G$ and $G / H$ is p-divisible. Then $H$ is p-pure in $G$.

Proof. Let $l$ be a $p$-imbedding function for $H$ in $G$ and suppose

$$
x=p^{n} g \in H \cap p^{n} G .
$$

Choose $k \geq \max \{0, l(n)-n\}$ and write $g=p^{k} g_{1}+h$. Then

$$
x=p^{n+k} g_{1}+p^{n} h, \quad n+k \geq l(n),
$$

so

$$
x-p^{n} h=p^{n+k} g_{1} \in H \cap p^{l(n)} G \subseteq p^{n} H .
$$

Thus $x \in p^{n} H$, as required.

LEMMA 1.6. Let $H<_{p} G$ with minimal imbedding function $l$ and suppose $C$ is pure in $H$ with $H / C$ p-divisible. Then l is the minimal imbedding function for $C$.

Proof. By the remarks following Proposition $1.4, l$ is an imbedding function for $C$. Let $l^{\prime}$ be the minimal function, so that $l^{\prime} \leq l$. For $h \in H \cap p^{l^{\prime}(n)} G$ we may choose $h_{1} \in H$ with $p^{m} h_{1}-h \in C$, where $m=\max \left\{l^{\prime}(n), n\right\}$. Then

$$
p^{m} h_{1}-h \in C \cap p^{l^{\prime(n)}} G \subseteq p^{n} C,
$$

so $h \in p^{n} C+p^{m} H \subseteq p^{n} H$. This gives $l(n) \leq l^{\prime}(n)$, as required.

Proposition 1.7. Let $G$ be a p-group, $H$ a subgroup of $G$, and $C$ a basic subgroup of $H$. Then $H<G$ if and only if $C<G$. In this case $l_{C}=l_{H}$ and there exists a basic subgroup $B$ of $G$ such that $B \cap H=C$ and $B / C$ is basic in $G / C$. Moreover, if $p^{k} P \subseteq C \subseteq P$ for some pure subgroup $P$ of $B$, then $p^{k} P_{0} \subseteq H \subseteq P_{0}$ for some pure subgroup $P_{0}$ of $G$.

Proof. If $H<G$, then $C$, being pure in $H$, is imbedded in $G$, and (by Lemma 1.6) $C$ and $H$ have the same imbedding function. Conversely, if $C<G$, then $H / C$ is divisible, and hence pure in $G / C$. Thus, by Proposition 1.4(iv), $H<G$.

Now, since $H / C$ is divisible, we can write $G / C=H / C \oplus L / C$ for some subgroup $L$ of $G$. Choose $B_{1}$ such that $B_{1} / C$ is basic in $L / C$. Then $B_{1}$ is imbedded and dense in $G$, and hence is pure in $G$ by Lemma 1.5. Clearly, $B_{1} \cap$ $H=C$ and $B_{1} / C$ is a direct sum of cyclic groups. Finally, by a well-known theorem of Kovacs [1, Theorem 33.4], $C$ is contained in a basic subgroup $B$ of $B_{1}$, which clearly is also basic in $G$. We have $C \subseteq B \cap H \subseteq B_{1} \cap H=C$ and $B / C \subseteq B_{1} / C$, so $B / C$ is a direct sum of cyclic groups. 
Now suppose $P$ is pure in $B$ and $p^{k} P \subseteq C \subseteq P$ for some $k$. Then $p^{k}(H+P) \subseteq H \subseteq H+P$ and

$$
\frac{H+P}{P} \cong \frac{H}{H \cap P}=H / C
$$

since $C \subseteq H \cap P \subseteq H \cap B=C$, so $(H+P) / P$ is divisible and hence pure in $G / P$. But $P$ is pure in $G$, so $P_{0}=H+P$ is pure in $G$.

\section{The difference function}

By Proposition 1.7, one need look no further than direct sums of cyclic groups to find the most general difference functions arising from imbedded subgroups, at least for $p$-groups. In this section we establish a condition for a subgroup of a direct sum of cyclic $p$-groups to be imbedded and use it to construct imbedded subgroups with a wide variety of difference functions.

Let $B=\bigoplus\left\langle b_{i}\right\rangle$ be (for the sake of simplicity) a standard basic subgroup, so that $\left\langle b_{i}\right\rangle$ is cyclic with generator $b_{i}$ of order $p^{i}$. Any subgroup $H$ of $B$ is also a direct sum of cyclic groups so we may write $H=\bigoplus\left\langle a_{k}\right\rangle$. Denote the exponent of $a_{k}$ by $\rho(k)$. Let $a_{k}=\sum_{i} \alpha_{k i} b_{i}$ and let $\xi(k, i)$ be the exponent of the highest power of $p$ dividing $\alpha_{k i}$. Then $\rho(k)=\operatorname{Max}\left\{i-\xi(k, i) \mid \alpha_{k i} \neq 0\right\}$ and we have the following lemma. We write $\mu(n, m)=\operatorname{Min}\{n, m\}$ for integers $n$ and $m$.

LEMMA 2.1. With the notation above, $H$ is imbedded in $B$ with imbedding function $l$ if and only if the following conditions holds: for any sequence $\left\{\chi_{k}\right\}$ of integers almost all of which are zero,

$$
p^{\mu(i, l(n))} \mid \sum_{k} \chi_{k} \alpha_{k i} \text { for all } i
$$

implies

$$
p^{\mu(\rho(k), n)} \mid \chi_{k} \quad \text { for all } k
$$

Proof. Divisibility condition $(*)$ simply expresses the fact that the element $x=\sum_{k} \chi_{k} a_{k}$ of $H$ lies in $p^{l(n)} B$, while condition (**) is equivalent to $x \in p^{n} H$.

To state our condition, we need one further bit of notation:

$$
L_{k}=\left\{i \mid \alpha_{k i} \neq 0\right\} ; \quad \lambda(k)=\sup L_{k} .
$$

In the following proof we use the easily verified fact that, to show that a subgroup is imbedded, it suffices to define an imbedding function on an unbounded set of integers. 
Proposition 2.2. With the notation above, $H$ is imbedded in $B$ with minimal difference function satisfying

$$
\delta_{\rho(k)-1}=\xi(k, \lambda(k)) \text { for all } k
$$

provided that

(1) if $j \neq k$ and $\lambda(j) \leq \lambda(k)$, then $\lambda(j)<\inf L_{k}$;

(2) for each $k, \xi(k, i)=0$ for some $i \in L_{k}$;

(3) for each $k, \rho(k) \geq i-\xi(k, i)+2$ for all $i \in L_{k}$, $i<\lambda(k)$.

Proof. Condition (3) and $\rho(k)=\operatorname{Max}\left\{i-\xi(k, i) \mid i \in L_{k}\right\}$ imply that $\rho(k)=\lambda(k)-\xi(k, \lambda(k))$. By (1), the sets $L_{k}$ are mutually disjoint. hence, (2) and (3) imply that $\{\rho(k) \mid k \in N\}$ is an unbounded set of integers, so $\{\rho(k)-1 \mid k \in N\}$ is an unbounded set of integers, so $\{\rho(k)-1 \mid k \in N\}$ is also. Now fix $j$, and let $n=\rho(j)=1, \delta_{n}=\xi(j, \lambda(j))$. Because the $L_{k}$ are mutually disjoint, (*) of Lemma 2.1 becomes, with $l(n)=n+\delta_{n}$,

$$
p^{\mu\left(i, n+\delta_{n}\right)} \mid \chi_{k} \alpha_{k i}, \quad \text { for all } i \in L_{k} .
$$

By definition of $\xi(k, i)$, this implies

$$
p^{\mu\left(i, n+\delta_{n}\right)-\xi(k, i)} \mid \chi_{k}, \quad \text { for all } i \in L_{k} .
$$

Now, if $\lambda(k) \leq n+\delta_{n}$, then $\mu\left(i, n+\delta_{n}\right)=i$ for all $i \in L_{k}$ and we obtain

$$
p^{i-\xi(k, i)} \mid \chi_{k}, \quad \text { for all } i \in L_{k} .
$$

In particular, $i=\lambda(k)$ yields $p^{\rho(k)} \mid \chi_{k}$, so $p^{\mu(\rho(k), n)} \mid \chi_{k}$, as required.

Suppose now that $k$ is such that $i \leq n+\delta_{n} \leq \lambda(k)$ for some $i \in L_{k}$. Recalling that

$$
n+\delta_{n}=\rho(j)-1+\xi(j, \lambda(j))=\lambda(j)-1,
$$

we have

$$
i \leq \lambda(j)-1 \leq \lambda(k), \quad \text { for all } i \in L_{k} .
$$

Now (1) implies $j=k$. Taking $i=\lambda(k)=\lambda(j)$ in $(*)^{\prime}$ yields

$$
p^{n+\delta_{n}-\xi(k, \lambda(k))} \mid \chi_{k}
$$

Since $j=k$ and $\delta_{n}=\xi(j, \lambda(j))$, this implies $p^{n} \mid \chi_{k}$.

Finally, for $k$ such that $n+\delta_{n}<\inf L_{k},(*)^{\prime}$ yields

$$
p^{n+\delta_{n}-\xi(k, i)} \mid \chi_{k}, \quad \text { for all } i \in L_{k} \text {. }
$$

By (2), for some $i, \xi(k, i)=0$, so $p^{n+\delta_{n}} \mid \chi_{k}$, so again $p^{n} \mid \chi_{k}$.

We have thus shown that $H$ is imbedded in $B$ and the minimal difference function evaluated at $\rho(j)-1$ does not exceed $\xi(j, \lambda(j))$. To complete the 
proof, we show that $x=p^{\rho(j)-2} a_{j}$, clearly not an element of $p^{\rho(j)-1} H$, is, however, in $p^{n+\delta_{n}-1} B=p^{\lambda(j)-2} B$ :

$$
\begin{aligned}
x & =p^{\rho(j)-2} a_{j}=\sum_{i \in L_{j}} p^{\rho(j)-2} \alpha_{j i} b_{i} \\
& =\sum_{i \in L_{j}} p^{\rho(j)-2+\xi(j, i)} \bar{\alpha}_{j i} b_{i},
\end{aligned}
$$

where $\alpha_{j i}=p^{\xi(j, i)} \bar{\alpha}_{j i}$. It suffices to show that

$$
\rho(j)-2+\xi(j, i) \geq \mu(l(j)-2, i) \text { for all } i \in L_{j},
$$

and this is guaranteed by (3).

More general conditions could be stated (at the expense of a certain amount of notational simplicity) but we feel that the ones given in 2.2 are general enough to provide insight into the following theorem, yet simple enough to handle conveniently.

THEOREM 2.3. Let $B$ be a standard basic subgroup and let $\left\{d_{n}\right\}$ be any sequence of non-negative integers. Then there exists an imbedded subgroup $H$ of $B$ whose minimal difference function $\delta$ has $\left\{d_{n}\right\}$ as a subsequence.

Proof. Define $\lambda(0)=0, \lambda(k)=d_{k}+\lambda(k-1)+3, k \geq 1$ (actually, any sequence $\lambda(n)$ with $\lambda(n+1)-\lambda(n)>2$ would suffice), and put

$$
L_{k}=\{\lambda(k-1)+1, \lambda(k)\}, \quad a_{k}=b_{\lambda(k-1)+1}+p^{d_{k}} b_{\lambda(k)},
$$

where $\left\{b_{i}\right\}$ is a basis for $B$. Clearly, $\left\{a_{k}\right\}$ forms a basis for the subgroup $H$ it generates, and

$$
\rho(k)=\lambda(k)-d_{k}=(\lambda(k-1)+1)+2,
$$

so (3) of Proposition 2.2 holds. Conditions (1) and (2) are clear.

It is not true that an arbitrary sequence of non-negative integers will itself be a minimal difference function for some imbedded subgroup. In fact, if $l$ is a minimal imbedding function for $H<G$, then

$$
H \cap p^{l(n+1)} G \subseteq p^{n+1} H \subseteq p^{n} H
$$

implies $l(n) \leq l(n+1)$, so the corresponding difference function must satisfy $\delta(n+1) \geq \delta(n)-1$ for every $n$. Thus, it is natural to ask: if $\left\{d_{n}\right\}$ is any sequence of non-negative integers satisfying $d_{n+1} \geq d_{n}-1$ for every $n$, does there exist a group with an imbedded subgroup whose minimal difference function is $\left\{d_{n}\right\}$ ? Theorem 2.4 answers this question affirmatively, and in fact shows that all these different varieties of imbedded subgroups may be accommodated within a single countable group. 
THEOREM 2.4. There exists a countable group $B$ with the following property: if $\left\{d_{n}\right\}$ is any sequence of non-negative integers satisfying $d_{n+1} \geq d_{n}-1$ for every $n$, then there is an imbedded subgroup $H$ of $B$ whose minimal difference function $\delta$ is given by $\delta(n)=d_{n}$ for every $n$.

Proof. Let $B_{i}=\bigoplus_{n=1}^{\infty}\langle x(i, n)\rangle$, where $\alpha(x(i, n))=p^{n}$, and let $B=$ $\bigoplus_{i=1}^{\infty} B_{i}$. Clearly, $B$ is countable. Given the sequence $\left\{d_{n}\right\}$ of non-negative integers satisfying $d_{n+1} \geq d_{n}-1$ for all $n$, define

$$
h_{i}=x(i, i-1)+p^{d_{i}} x\left(i, i+d_{i}\right), \quad i=1,2, \ldots,
$$

where $x(1,0)=0$. Let $H \subseteq B$ be the sum of the subgroups $\left\langle h_{i}\right\rangle$ for all $i$, and observe that this sum is direct. We must show that, for all $k, H \cap p^{k+d_{k}} B \subseteq$ $p^{k} H$ but $H \cap p^{k+d_{k}-1} B \nsubseteq p^{k} H$.

Let $h \in H \cap p^{k+d_{k}} B$. Then

$$
\begin{aligned}
h= & a_{1} p^{d_{1}} x\left(1,1+d_{1}\right)+a_{2}\left(x(2,1)+p^{d_{2}} x\left(2,2+d_{2}\right)\right) \\
& +\cdots+a_{i}\left(x(i, i-1)+p^{d_{i}} x\left(i, i+d_{i}\right)\right)+\cdots,
\end{aligned}
$$

where $a_{1}, a_{2}, \ldots, a_{i}, \ldots$ are integers, and there exists $b \in B$ such that $p^{k+d_{k}} b=$ $h$. Thus, $p^{k+d_{k}}$ must divide each summand; that is, for each $i$ there are integers $q, r, s, t$ such that

(1) $a_{i}=q p^{k+d_{k}}+r p^{i-1}$ and

(2) $a_{i} p^{d_{i}}=s p^{k+d_{k}}+t p^{i+d_{i}}$.

Now, if $i \leq k$, then $i+d_{i} \leq k+d_{k}$ by hypothesis (since an easy induction argument yields $d_{k} \geq d_{k-(k-i)}-(k-i)$ for each such $\left.i\right)$, so $p^{i}$ divides $a_{i}$ by (2), implying

$$
a_{i}\left(x(i, i-1)+p^{d_{i}} x\left(i, i+d_{i}\right)\right)=0 .
$$

If $i>k$, then $p^{k}$ divides $a_{i}$ by (1). Hence, $h \in p^{k} H$.

Finally, for the direct summand

$$
\left\langle h_{k}\right\rangle=\left\langle x(k, k-1)+p^{d_{k}} x\left(k, k+d_{k}\right)\right\rangle
$$

of $H$, we have $p^{k-1} h_{k}=p^{k+d_{k}-1} x(k)_{k+d_{k}} \in p^{k+d_{k}-1} B$, but $p^{k-1} h_{k} \notin p^{k} H$ because $p^{k}\left\langle h_{k}\right\rangle=0$.

Theorem 2.4 does not completely replace Theorem 2.3 , in that 2.3 applies to a single standard basic subgroup, whereas 2.4 requires a countable sum of standard basic subgroups. This suggests the question: given a particular direct sum of cyclic groups $G$, what additional restrictions must be imposed on a sequence of non-negative integers in order that it describe the minimal difference function of some imbedded subgroup of $G$ ? 


\section{Some trivially imbedded subgroups}

We have remarked that if $G$ is a $p$-group, $P$ is pure in $G$, and there exists $k$ such that $p^{k} P \subseteq H \subseteq P$ for some subgroup $H$ of $G$, then $H$ is imbedded in $G$. To state the general version of this fact, we say a group is p-locally bounded if its $p$-primary component is bounded.

Proposition 3.1. Let $H$ be a subgroup of a group $G$ and let $M$ be a p-pure subgroup of $G$ containing $H$ with $M / H$ p-locally bounded. If $p^{m}$ is a bound for $(M / H)_{p}$, then

$$
H \cap p^{n+m} G=p^{n}\left(H \cap p^{m} G\right) \text { for all } n .
$$

In particular, $H<_{p} G$ with bounded difference function.

Proof. It suffices to prove that $H \cap p^{n+m} G \subseteq p^{n}\left(H \cap p^{m} G\right)$. Let $x \in H \cap$ $p^{n+m} G=H \cap p^{n+m} M$. Then $x=p^{n+m} y$, for some $y \in M$, so $y+H \in(M / H)_{p}$. But then $p^{m} y \in H \cap p^{m} G$, so $x \in p^{n}\left(H \cap p^{m} G\right)$, as required.

We shall eventually see that Proposition 3.1 has a converse (Theorem 5.4). It seems surprising that the existence of a pure subgroup $P$ of $B$ such that $p^{k} P \subseteq H \subseteq P$ is not equivalent to $H$ being imbedded with a bounded difference function. In fact, we have

THEOREM 3.2. The subgroup $H$ of the standard basic subgroup $B$ constructed in Theorem 2.3 satisfies $p^{m} P \subseteq H \subseteq P$ for some pure subgroup $P$ of $B$ and some integer $m$ if and only if $\delta(n)=0$ for all sufficiently large $n$.

Proof. In 2.3 we defined $\lambda(k)=d_{k}+\lambda(k-1)+3$ with $\lambda(0)=0$, so by induction $\lambda(k)=3 k+\sum_{i=1}^{k} d_{i}$. The subgroup $H$ had elements $a_{k}=$ $b_{\lambda(k-1)+1}+p^{d_{k}} b_{\lambda(k)}$ as basis and, because

$$
S=\sum_{k}\left\langle b_{\lambda(k-1)+1}\right\rangle+\sum_{k}\left\langle b_{\lambda(k)}\right\rangle
$$

is a summand of $B$ containing $H$, we may restrict attention to $S$.

Now suppose $x \in S$ and $p^{t} x \in H$. Let $x=\sum c_{i} b_{i}$, where $i$ ranges over the integers of the forms $\lambda(k-1)+1$ and $\lambda(k)$. Then

$$
p^{t} x=\sum_{k} p^{t} c_{\lambda(k-1)+1} b_{\lambda(k-1)+1}+\sum_{k} p^{t} c_{\lambda(k)} b_{\lambda(k)} .
$$

But $p^{\imath} x \in H$, so

$$
p^{t} x=\sum_{n} \zeta_{n} a_{n}=\sum_{n} \zeta_{n} b_{\lambda(n-1)+1}+p^{d_{n}} \zeta_{n} b_{\lambda(n)}
$$


Thus, $p^{t} c_{\lambda(k-1)+1} \equiv \zeta_{k}\left(\bmod p^{\lambda(k-1)+1}\right)$ and $p^{t} c_{\lambda(k)} \equiv p^{d_{k}} \zeta_{k}\left(\bmod p^{\lambda(k)}\right)$ for all $k$. From the first congruence we obtain

$$
p^{t+d_{k}} c_{\lambda(k-1)+1}-p^{d_{k}} \zeta_{k}=p^{\lambda(k-1)+1+d_{k}} q,
$$

and from the second,

$$
p^{d_{k}} \zeta_{k}=p^{t} c_{\lambda(k)}-p^{\lambda(k)} q^{\prime}
$$

so

$$
\begin{aligned}
p^{t+d_{k}} c_{\lambda(k-1)+1}-p^{t} c_{\lambda(k)} & =p^{\lambda(k-1)+1+d_{k}} q-p^{\lambda(k)} q^{\prime} \\
& =p^{\lambda(k-1)+1+d_{k}} r_{k} .
\end{aligned}
$$

Using this value for $p^{t} c_{\lambda(k)}$ we obtain

$$
\begin{aligned}
p^{t} x= & \sum_{k} p^{t} c_{\lambda(k-1)+1} b_{\lambda(k-1)+1} \\
& +\sum_{k} p^{t+d_{k}} C_{\lambda(k-1)+1} b_{\lambda(k)}-\sum_{k} p^{\lambda(k-1)+1+d_{k}} r_{k} b_{\lambda(k)} \\
= & \sum_{k} p^{t} c_{\lambda(k-1)+1} a_{k}-\sum_{k} r_{k} p^{\lambda(k-1)+1+d_{k}} b_{\lambda(k)} .
\end{aligned}
$$

Now,

$$
p^{\lambda(k-1)+1+d_{k}} b_{\lambda(k)}=p^{\lambda(k-1)+1} a_{\lambda(k)}
$$

so finally we have

$$
p^{t} x=p^{t}\left(\sum_{k} c_{\lambda(k-1)+1} a_{k}\right)-\sum_{k} r_{k} p^{\lambda(k-1)+1} a_{k} .
$$

Moreover, $p^{\lambda(k-1)+1} a_{k}$ has order $p^{2}$ for each $k$.

Thus, we have shown that

$$
H \cap p^{t} S \subseteq p^{t} H+H\left[p^{2}\right]
$$

for each $t$. Suppose now that $H \cap p^{n+m} B \subseteq p^{n}\left(H \cap p^{m} B\right)$, which by 3.1 would be implied by the existence of a pure subgroup $P$ of $B$ with $p^{m} P \subseteq H \subseteq P$. Then $H \cap p^{n+m} S=H \cap S \cap p^{n+m} B=H \cap p^{n+m} B$ and $H \cap p^{m} B=H \cap S \cap p^{m} B=$ $H \cap p^{m} S$, so a similar situation holds for $S$ in place of $B$. For $n \geq 2$, we obtain

$$
H \cap p^{n+m} S \subseteq p^{n}\left(H \cap p^{m} S\right) \subseteq p^{n}\left(p^{m} H+H\left[p^{2}\right]\right)=p^{n+m} H ;
$$

that is, $H \cap p^{t} S \subseteq p^{t} H$ for $t>m+1$. Clearly, this yields $\delta(t)=0$ for $t>m+1$.

Conversely, suppose $\delta(t)=0$ for $t>m$, so that $H \cap p^{t} B \subseteq p^{t} H$ for $t>m$. Let $P=\sum_{k>m}\left\langle a_{k}\right\rangle$. Since $a_{k}=b_{\lambda(k-1)+1}+b_{\lambda(k)}$ for $k>m, P$ is clearly pure in $B$. The $H / P$ is bounded and imbedded in $B / P$, hence is discrete, so there exists a bounded pure subgroup $M / P$ of $B / P$ containing $H / P$ (for example, 
if $H / P \cap p^{i} B / P=0$, let $M / P$ be $p^{i}$-high in $B / P$ containing $\left.H / P\right)$. Then $M$ satisfies the conditions of the theorem.

It should be mentioned that, for $p$-groups $G$, subgroups $H$ satisfying $p^{m} H$ $\subseteq P \subseteq H$ with $P$ pure in $G$ need not be imbedded since $H / P$ need not be discrete in $G / P$. One can show that such a subgroup $H$ is imbedded if and only if $H \cap p^{i} G=p^{i} H$ for all large $i$. This condition on $H$ is in fact equivalent to the existence of a pure subgroup $P$ as above.

\section{Regular imbeddedness and kernels of purity}

Section 3 suggests the following definitions.

Definitions 4.1. A subgroup $H$ of a group $G$ is $m$-regularly p-imbedded, where $m$ is a non-negative integer and $p$ is a prime, provided that $H \cap$ $p^{m+n} G=p^{n}\left(H \cap p^{m} G\right)$ for all $n$. We say that $H$ is regularly p-imbedded if $H$ is $m$-regularly $p$-imbedded for some $m$, and that $H$ is regularly imbedded if $H$ is regularly $p$-imbedded for all $p$.

Our aim here is to establish a relation between regularly imbedded subgroups and kernels of purity. This will be applied in the next section to characterize regularly imbedded subgroups. First we need several lemmas.

Lemma 4.2. Let $H$ be a subgroup of $G$ and let $N$ be a minimal neat subgroup of $G$ containing $H$. If, for some prime $p$ and integer $n, G[p]=$ $H[p]+p^{n} G[p]$, then $N \cap p^{t} G=p^{t} N$ for all $t \leq n+1$.

Proof. Our hypotheses yield $H[p]=N[p]$ and $N \cap p G=p N$. Assume that $N \cap p^{t} G=p^{t} N$ for some $t \leq n$ and let $x \in N \cap p^{t+1} G$. then $x=p^{t+1} g=p y$, $y \in N$, so $y-p^{t} g \in G[p]=N[p]+\left(p^{t} G\right)[p]$. If $y-p^{t} g=z+p^{t} w$ with $z \in N[p]$ and $p^{t} w \in\left(p^{t} G\right)[p]$, then $y-z=p^{t}(g+w) \in N \cap p^{t} G=p^{t} N$. Hence, $x=p(y-z) \in p^{t+1} N$, as required.

LEMMA 4.3. Let $H$ be $m$-regularly $p$-imbedded in $G$ and let $N$ be a minimal neat subgroup containing $H$. If $N \cap p^{t} G=p^{t} N$ for every $t \leq m$, then $N$ is p-pure in $G$.

Proof. We must show that if $k \geq 1$ and $x \in N \cap p^{m+k} G$, then $x \in p^{m+k} N$. We may assume $x \neq 0$; so $\langle x\rangle \cap H \neq 0$, since $H$ is essential in $N$. Thus, there is a smallest positive integer $c_{x}$ satisfying $c_{x} x \in H$. We use induction on $c_{x}$. If $c_{x}=1$, then $x \in H$; so $x \in H \cap p^{m+k} G=p^{k}\left(H \cap p^{m} G\right) \subseteq p^{k}\left(N \cap p^{m} G\right)=$ $p^{k+m} N$. For the induction hypothesis we assume that every $x \in N \cap p^{m+k} G$ 
with $c_{x}<n$ satisfies $x \in p^{m+k} N$. Let $x \in N \cap p^{m+k} G$ with $c_{x}=n$, and let $n=q p^{j}$ with $(q, p)=1$. Then

$$
n x \in H \cap p^{j+m+k} G=p^{j+k}\left(H \cap p^{m} G\right) \subseteq p^{j+k}\left(N \cap p^{m} G\right)=p^{j+k+m} N .
$$

There exist integers $r$ and $s$ such that $1=r q+s p^{m+k}$, so

$$
p^{j} x=\left(r n x+s p^{m+k+j} x\right) \in p^{m+k+j} N .
$$

Thus, there exists $y \in N$, as desired; otherwise, $p^{j-1}\left(x-p^{m+k} y\right) \in N[p] \subseteq H$ and $p^{j-1}<n$, so $x-p^{m+k} y \in p^{m+k} N$ by the induction hypothesis. Therefore, $x \in p^{m+k} N$.

LeMMA 4.4. Let $H$ be a kernel of purity in $G$ and let $p$ be a prime. If $m \geq 0$ and $H \cap p^{m+1} G \nsubseteq p H$, then $G[p]=H[p]+\left(p^{m} G\right)[p]$.

Proof. Let $h=p^{m+1} g \in H \cap p^{m+1} G$ with $h \notin p H$. then $p^{m} g+z \notin H$ for every $z \in G[p]$. Let $x \in G[p]$ and let $y=x+p^{m} g$. Let $K=\langle y, H\rangle$ and let $N$ be minimal neat in $G$ containing $K$. Then $K$ is essential in $N$ and, since $y \notin H$ and $p y \in H, H$ is also essential in $N$. Thus, $N$ is minimal neat containing $H$, so $N$ is pure. Hence there exists $w \in N$ such that $p^{m+1} g=p^{m+1} w$, so

$$
p^{m} g-p^{m} w \in\left(p^{m} G\right)[p] .
$$

Thus, $y-p^{m} w=x+p^{m} g-p^{m} w \in N \cap G[p]=N[p]=H[p]$, so $x \in$ $H[p]+\left(p^{m} G\right)[p]$. Therefore, $G[p]=H[p]+\left(p^{m} G\right)[p]$.

THEOREM 4.5. A subgroup $H$ of $G$ is a kernel of purity if and only if $H$ satisfies either (1) or (2) for each prime $p$ :

(1) $H[p]$ is dense in $G[p]$ (in the p-adic topology of $G$ ) (compare [5]).

(2) There exists an integer $m \geq 1$ such that

(a) $G[p]=H[p]+p^{m-1} G[p]$, and

(b) $H$ is $m$-regularly p-imbedded in $G$.

Proof. Let $N$ be minimal neat containing $H$ and let $p$ be a prime. If (1) holds, then $N$ is $p$-pure by 4.2 . If (2) holds, then (a) and 4.2 imply $N \cap p^{t} G=p^{t} N$ for $t \leq m$. Then (b) and 4.3 imply $N$ is $p$-pure. Thus, $H$ is a kernel of purity.

Conversely, assume $H$ is a kernel of purity and $p$ is a prime. If (1) does not hold, let $m$ be the least positive integer such that $G[p] \neq H[p]+\left(p^{m} G\right)[p]$. Let $N$ be minimal neat containing $H$ and let $x \in H \cap p^{m+1} G$. Lemma 4.4 implies $x=p h, h \in H$, and the purity of $N$ implies $x=p^{m+1} y, y \in N$. Thus $h-p^{m} y \in N[p]=H[p]$, so there exists $z \in H[p]$ such that $h-z \in H \cap p^{m} G$. Therefore, $x=p(h-z) \in p\left(H \cap p^{m} G\right)$, proving that $H \cap p^{m+n} G=p^{n}\left(H \cap p^{m} G\right)$ 
for $n=1$. A routine induction argument completes the proof for $n>1$. Thus, $H$ is $m$-regularly $p$-imbedded, so (2) holds.

\section{Regularly imbedded subgroups}

We can now characterize regularly imbedded subgroups as those which are "approximately pure." We need a few lemmas.

LEMMA 5.1. Let $H$ be a subgroup of $G$. For any prime $p$ and nonnegative integer $m$, there exists a decomposition

$$
G[p]=A \oplus B \oplus C \oplus D
$$

such that

(1) $H[p]=B \oplus C$,

(2) $p^{m-1} G[p]=C \oplus D$, and

(3) $A \cap p^{m-1} G=0$.

Proof. Let $C=H \cap\left(p^{m-1} G\right)[p]$. Let $D$ be a complement of $C$ in $\left(p^{m-1} G\right)[p]$. Then $\left(p^{m-1} G\right)[p]=C \oplus D$. Let $B$ be a complement of $C$ in $H[p]$. Then $H[p]=B \oplus C$ and $H[p]+\left(p^{m-1} G\right)[p]=B \oplus C \oplus D$. Finally, let $A$ be a complement of $H[p]+\left(p^{m-1} G\right)[p]$ in $G[p]$, so that $G[p]=A \oplus B \oplus C \oplus D$ and $A \cap p^{m-1} G=0$.

Lemma 5.2. Let $H$ be $m_{i}$-regularly $p_{i}$-imbedded in $G$ for every prime $p_{i}$. There exists a kernel of purity $K$ such that $H \subseteq K, K / H$ is a torsion group, and $K[p] \cap p_{i}^{m_{i}-1} G \subseteq H$ for every $i$.

Proof. For each prime $p_{i}$ consider the decomposition $G\left[p_{i}\right]=A_{i} \oplus B_{i} \oplus$ $C_{i} \oplus D_{i}$ given by 5.1. Let $K=H \oplus \sum_{1}^{\infty} A_{k}$. It is easy to check that $K\left[p_{i}\right] \cap$ $p_{i}^{m_{i}-1} G \subseteq H$ for every $i$. Let $x \in K \cap p_{i}^{m_{i}+n} G$. Then $x=h+\sum_{1}^{N} a_{k}$, for $h \in H$ and $a_{k} \in A_{k}$. Now, $\sum_{k \neq i} a_{k} \in p_{i}^{n}\left(K \cap p_{i}^{m_{i}} G\right)$, implying $\left(h+a_{i}\right) \in p_{i}^{m_{i}+n} G$. Thus, $p_{i} h \in H \cap p_{i}^{m_{i}+n+1} G=p_{i}^{n+1}\left(H \cap p_{i}^{m_{i}} G\right)$. Let $h_{1} \in H \cap p_{i}^{m_{i}} G$ such that $p_{i} h=p_{i}^{n+1} h_{1}$. Then $h-p_{i}^{n} h_{1}=h_{2} \in H\left[p_{i}\right]$, so $h_{2}+a_{i}=\left(h+a_{i}\right)-p_{i}^{n} h_{1} \in$ $\left(p_{i}^{m_{i}+n} G\right)\left[p_{i}\right]$. It follows from the construction of $A_{i}$ that $a_{i}=0$. Hence, $h \in H \cap p_{i}^{m_{i}+n} G=p_{i}^{n}\left(H \cap p_{i}^{m_{i}} G\right)$, and $x=h+\sum_{1}^{N} a_{k} \in p_{i}^{n}\left(K \cap p_{i}^{m_{i}} G\right)$. Thus, $K \cap p_{i}^{m_{i}+n} G=p_{i}^{n}\left(K \cap p_{i}^{m_{i}} G\right)$, so $K$ is $m_{i}$-regularly $p_{i}$-imbedded. Also, it is clear that $G\left[p_{i}\right]=K\left[p_{i}\right]+\left(p_{i}^{m_{i}-1} G\right)\left[p_{i}\right]$. Therefore, by $4.5, K$ is a kernel of purity. 
LEMMA 5.3. Let $l(n)$ be a p-imbedding function for $H$ in $G$ satisfying $l(n) \geq$ $n$, for all $n$. If $\left(p^{k} G\right)[p] \subseteq H$, then $p^{l(k+1)} G_{p} \subseteq H$.

Proof. We use induction on $n$ to show that $\left(p^{l(k+1)} G\right)\left[p^{n}\right] \subseteq H$ for every $n \geq 1$. The case $n=1$ follows from the hypothesis. Thus, assume $\left(p^{l(k+1)} G\right)\left[p^{n}\right] \subseteq H$ and let $x \in\left(p^{l(k+1)} G\right)\left[p^{n+1}\right]$. Then $p x \in H \cap p^{l(k+1)} G \subseteq$ $p^{k+1} H$, so there exists $y \in H$ such that $x-p^{k} y \in\left(p^{k} G\right)[p] \subseteq H$. Thus $x \in H$ and $\left(p^{l(k+1)} G\right)\left[p^{n+1}\right] \subseteq H$, as desired.

THEOREM 5.4. A subgroup $H$ is regularly imbedded in $G$ if and only if there exists a pure subgroup $M$ such that $H \subseteq M$ and $M / H$ is a locally bounded torsion group.

Proof. Assume $H$ is regularly imbedded in $G$; say $H$ is $m$-regularly $p$ imbedded for the prime $p$. By 5.2 there exists a kernel of purity $K$ such that $K / H$ is a torsion group and $K[p] \cap p^{m-1} G \subseteq H$. Let $M$ be minimal neat containing $K$. Then $M$ is pure and $M[p]=K[p]$. Thus $\left(p^{m-1} M\right)[p]=$ $M[p] \cap p^{m-1} G=K[p] \cap p^{m-1} G \subseteq H$. Since $M$ is pure, $H$ is also $m$-regularly $p$ imbedded in $M$, so $p^{2 m} M_{p} \subseteq H$ by 5.3. Now, from the fact that $l(n)=n+m$ is an imbedding function for $H$ in $M$, it follows that $(M / H)_{p}$ is bounded by $p^{3 m}$. Finally, $M / H$ is torsion, since $K / H$ is torsion and $M / K$ is torsion.

The converse follows from Proposition 3.1.

\section{A Serre class}

In this section we determine those groups all of whose subgroups are imbedded.

Proposition 6.1. The following are equivalent:

(1) Every subgroup of $G$ is imbedded;

(2) every homomorphic image of $G$ is locally bounded;

(3) every homomorphic image of $G$ is reduced.

Proof. Suppose (1) holds and let $G / K$ be an image of $G$. Then $K$ and $H=\{x \in G \mid p x \in K\}$ are both imbedded in $G$, so $H / K=(G / K)[p]$ is imbedded in $G / K$, and hence is discrete. This yields (2).

The existence of a non-reduced image of $G$ implies the existence of a nonzero divisible image, hence of a torsion divisible image, which cannot be locally bounded. Thus, (2) implies (3). 
Now assume (3) and let $H$ be an arbitrary image of $G$. If $H_{p}$ is unbounded form some $p$, then $H_{p}$ contains a proper basic subgroup $B$ and $H_{p} / B$ is nonzero and divisible. Hence, $H_{p} / B$ is a summand of $H / B$, so it is an image of $H / B$, so also of $G$. Thus, (2) holds.

Finally, (2) obviously implies (1), by Proposition 3.1.

Denote by $\mathscr{C}$ the class of abelian groups all of whose subgroups are imbedded. Thus, $G \in \mathscr{C}$ if and only if $G$ has no non-zero divisible images.

Theorem 6.2. Let $0 \rightarrow A \rightarrow B \rightarrow C \rightarrow 0$ be exact. Then $B \in \mathscr{C}$ if and only if $A$ and $C$ belong to $\mathscr{C}$.

Proof. If $B \in \mathscr{C}$, since any image of $C$ is an image of $B, C \in \mathscr{C}$. On the other hand, if $A \rightarrow D \rightarrow 0$ is exact with $D$ divisible, then

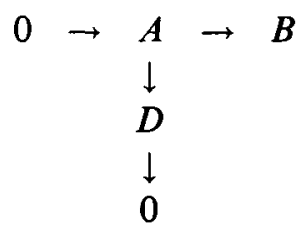

yields $B \rightarrow D \rightarrow 0$. Thus $D=0$ and $A \in \mathscr{C}$.

Conversely, suppose $A, C \in \mathscr{C}$ and let $0 \rightarrow K \rightarrow B \rightarrow D \rightarrow 0$ be exact with $D$ divisible. Then $B /(A+K)$ (taking the monomorphisms to be inclusions) is an image of both $C \cong B / A$ and $D \cong B / K$, so it is a divisible image of $C$. Since $C \in \mathscr{C}$ this gives $B=A+K$ and we have

$$
D=\frac{B}{K}=\frac{A+K}{K}=\frac{A}{A \cap K} .
$$

But $A \in \mathscr{C}$, so $D=0$.

By the theorem above, to characterize the elements of $\mathscr{E}$ it suffices to characterize the torsion groups in $\mathscr{C}$ (and these are simply the locally bounded torsion groups) and the torsion free elements of $\mathscr{C}$. Clearly, every finitely generated group is in $\mathscr{C}$, and a free abelian group is in $\mathscr{C}$ if and only if it has finite rank. For the general torsion free case we have

THEOREM 6.3. A torsion free group $G$ is in $\mathscr{C}$ if and only if $G$ is a subdirect sum of finitely many rank 1 groups the types of which contain no $\infty$ 's.

Proof. Clearly, a rank 1 torsion free group whose type has no $\infty$ 's is in $\mathscr{C}$; hence (by induction using Theorem 6.2), any finite direct sum of such groups is in $\mathscr{C}$; hence (by 6.2 again), any subgroup of such a direct sum is in $\mathscr{C}$. 
Conversely, let $G$ be a torsion free group in $\mathscr{C}$ and let $X$ be a maximal independent set in $G$. Then the free group on $X$ is contained in $G$, hence is in $\mathscr{C}$, so has finite rank by our remark above. Thus, $G$ has finite rank. Let $L_{x}$ be the pure subgroup of $G$ generated by $\{y \in X \mid y \neq x\}$ for $x \in X$, and let $\varphi_{x}: G \rightarrow G / L_{x}$ be the natural map. Then $G / L_{x}$ is rank 1 and in $\mathscr{E}$ so its type is of the desired form. Finally, the map $\varphi: G \rightarrow \bigoplus G / L_{x}$ induced by the $\varphi_{x}$ has kernel $\bigcap_{x \in X} L_{x}=0$.

It is easy to see that in a torsion free group, every imbedded subgroup is regularly imbedded. Many characterizations of $p$-groups with bounded basic subgroups have been given, and it may be of interest that these groups also are precisely the $p$-groups in which every imbedded subgroup is regularly imbedded. Slightly more generally, we have

Proposition 6.4. For a torsion group $G$, every imbedded subgroup is regularly imbedded if and only if the reduced part of $G$ is locally bounded.

Proof. Any unbounded reduced p-group has, by Theorems 3.2 and 5.4, imbedded subgroups that are not regularly imbedded, so the necessity is clear. For sufficiency it is enough to treat the case of $p$-groups. Thus, let $G=D \oplus R$ with $D$ divisible and $R$ reduced and suppose $R$ is bounded. Then any basic subgroup of $G$ is bounded and the result follows from Propositions 1.6 and 3.1.

\section{Totally quasi-complete groups}

Despite the similarity in the definitions of purity and imbeddedness, Theorems 2.3 and 2.4 establish the existence of a wide variety of imbedded subgroups that are not pure. This fact led one of us (in [6]) to consider for imbeddedness a question originally posed for purity by Head in [3] and settled by Hill and Megibben in [4].

Definitions 7.1. A group $G$ is torsion complete if it is the torsion part of the $p$-adic completion of a direct sum of cyclic groups. $G$ is quasi-complete ( $l$-quasi-complete) if the closure of every pure ( $l$-imbedded) subgroup is itself pure ( $l$-imbedded); $G$ is totally quasi-complete if it is $l$-quasi-complete for every $l$.

Hill and Megibben showed that quasi-complete groups need not be torsion complete, although the reverse implication holds. It was shown in [6] that every torsion complete group is totally quasi-complete. That paper ends with the conjecture that an abelian $p$-group is torsion complete if and only if it 
is totally quasi-complete. However, we have subsequently found that this is not the case; a modification of Hill and Megibben's arguments in [4] yields the following theorem. For convenience, we first restate here a lemma from [6] that is needed in the proof.

Lemma 7.2 ([6], Corollary 2.8). Let $h$ be imbedded in $G$. Then $H^{-}$is imbedded if and only if $(G / H)^{1}$ is divisible.

LEMMA 7.3. Let $A$ be a separable p-group. The following are equivalent:

(a) $A$ is quasi-complete;

(b) $A$ is l-quasi-complete for some imbedding function l (greater than or equal to the identity function);

(c) $A$ is totally-quasi-complete.

Proof. It is clear that (c) implies (b), and (b) implies (a) because the identity function is an imbedding function for pure subgroups. To show (a) implies (c) we use Hill and Megibben's characterization of quasi-completeness ([4], Theorem 2).

A separable $p$-group $A$ is quasi-complete if and only if

$$
A[p]+S^{-}=\bar{B}[p]
$$

for every nondiscrete subsocle $S$ of $A$.

(Here $B$ denotes a basic subgroup of $A$, and closures are understood to be in $\bar{B}$, the torsion completion of $B$.)

Let $A$ be quasi-complete. If $G<A$ and $G$ is bounded, then $G$ is discrete and thus $G^{-}<A$. Hence, assume $G$ is unbounded and is $l$-imbedded in $A$, where $l(1)=k$. Consider $p^{k} a \in G^{-}$for some $a \in A$. $G^{-} / G=(\bar{B} / G)^{1}$, and, since $\bar{B}$ is totally quasi-complete, $G^{-}<\bar{B}$, so $(\bar{B} / G)^{1}$ is divisible by Lemma 7.2. Thus there exist $g \in G, h \in G^{-}$such that $p^{k} h=p^{k} a+g$. Now, $p^{k}(h-a)=p g^{\prime}$ for some $g^{\prime} \in G$ (since $A$ pure in $\bar{B}$ implies $G$ is $l$-imbedded in $\bar{B}$ ), so

$$
p^{k-1} h-\left(p^{k-1} a+g^{\prime}\right) \in \bar{B}[p]=A[p]+G^{-}[p]
$$

by (*). Hence, there exist $a^{\prime} \in A[p], h^{\prime} \in G^{-}[p]$ such that

$$
p^{k-1} h-h^{\prime}=p^{k-1} a+a^{\prime}+g^{\prime} \in G^{-} \cap A .
$$

Now, $p\left(p^{k-1} a+a^{\prime}+g^{\prime}\right)=p^{k} a+p g^{\prime}$, so $p^{k-1} a+a^{\prime}+g^{\prime}$ is a solution of $p x \equiv p^{k} a(\bmod G)$. Hence, any element of $(A / G)^{1}=\left(G^{-} \cap A\right) / G$ is divisible by $p$ in $\left(G^{-} \cap A\right) / G$, so

$$
\left(G^{-} \cap A\right) / G=p\left(\left(G^{-} \cap A\right) / G\right) .
$$


That is, $\left(G^{-} \cap A\right) / G$ is divisible and hence pure in $A / G$. Thus, by Proposition 1.4(iv) and the remark following it, $l$ is an imbedding function for $G^{-} \cap A$ in $G$.

\section{References}

[1] L. Fuchs, Infinite Abelian Groups, vol. I, Academic Press, New York, 1970.

[2] L. Fuchs, Infinite Abelian Groups, vol. II, Academic Press, New York, 1970.

[3] T. Head, 'Remarks on a problem in primary abelian groups,' Bull. Soc. Math. France 91 (1963), 109-112.

[4] P. Hill and C. Megibben, 'Quasi-closed primary groups,' Acta Math. Acad. Sci. Hungar. 16 (1965), 271-274.

[5] C. Megibben, 'Kernels of purity in abelian groups,' Publ. Math. Debrecen 11 (1964), 160-164.

[6] J. D. Moore, 'On quasi-complete abelian p-groups,' Rocky Mountain J. Math. 5 (1975), 601-609.

[7] J. D. Moore and H. Bowman, 'A note on the large subgroup topology for abelian groups,' Comment. Math. Univ. St. Paul 22:2 (1973), 7-17.

[8] J. D. Moore and H. Bowman, 'A method for obtaining proper classes of short exact sequences of abelian groups,' Publ. Math. Debrecen 24 (1977), 59-63.

Colby College

Waterville, Maine 04901

U.S.A.

Wesleyan University

Middletown, Connecticut 06457

U.S.A.
Arizona State University

Tempe, Arizona 85287

U.S.A. 